recent in vivo pathogenesis studies in mice, have shown that intestinal colonization by RG strains isolated from Lupus patients in flare, but not RG strains from healthy adults, induce zonulin-dependent increases in intestinal permeability, RG translocation to mesenteric lymph nodes as well as serum IgG anti-RG antibody and anti-native DNA autoantibody responses.

Conclusions As many Lupus patients are known to suffer relapsing illness despite appropriate treatment, we speculate that gut blooms of pathogenic bacteria that impair gut barrier function and stoke systemic inflammation directly contribute to immunopathogenesis. We propose that future therapeutic interventions designed to promote a durable remission consider the potential necessity to target both the immunologic abnormalities of the disease, as well as re-establish stability within the gut microbiota community.

Acknowledgments This work was supported in part by National Institutes of Health Grants; R01-AR42455, P50 AR070591, the Lupus Research Alliance, and the Judith and Stewart Colton Autoimmunity Center (GJS). 16S rRNA amplicon sequence determinations and analysis were supported by the P. Robert Majumder Charitable Trust.

\section{THE RISING INCIDENCE, PREVALENCE AND MORTALITY GAP OF LUPUS NEPHRITIS: A POPULATION-BASED STUDY OVER FOUR DECADES}

\begin{abstract}
1,2Mehmet Hocaoglu, 'Maria 0 Valenzuela-Almada, 'Jesse Y Dabit, 'Shirley-Ann OseiOnomah, ${ }^{1,3}$ Baptiste Chevet, ${ }^{4}$ Rachel E Giblon, ${ }^{1,4}$ Cynthia S Crowson, ${ }^{1,5}$ Alí Duarte-García*. ${ }^{1}$ Division of Rheumatology, Mayo Clinic, Rochester, MN, USA; ${ }^{2}$ Department of Medicine, University of Maryland Medical Center, Midtown Campus, Baltimore, MD, USA; ${ }^{3}$ Division of Rheumatology, Brest Teaching Hospital, Brest, France; LBAI, UMR1227, Univ Brest, Inserm, CHU de Brest, Brest, France; ${ }^{4}$ Department of Quantitative Health Sciences, Mayo Clinic, Rochester, MN, USA; ${ }^{5}$ Division of Nephrology and Hypertension, Mayo Clinic, Rochester, MN, USA
\end{abstract}

\subsection{6/lupus-2021-lupus21century.30}

Background There is paucity of population-based studies that investigate the epidemiology of lupus nephritis (LN) and its long-term outcomes.

Methods We used the Rochester Epidemiology Project (REP) a record-linkage system that includes all residents from Olmsted County, Minnesota since 1966 with additional counties since 2010. A cohort of LN patients between 1976 and 2018 in an eight-county region was identified based on (1) biopsy proven LN in the presence of a positive ANA or ds-DNA antibody, or (2) meeting ACR/EULAR 2019 criteria and having documented proteinuria $(500 \mathrm{mg}$ in $24 \mathrm{hrs}$ urine collection or protein/creatinine ratio $>0.5)$ that did not have a better explanation than SLE. Age- and sex-specific incidence rates and point prevalence for four decades, adjusted to 2000 US total population, were reported. Standardized mortality ratio (SMR), survival rates, and time to end-stage renal disease (ESRD) were estimated using Kaplan-Meier methods. Poisson regression models were used to look at trends in relative survival over time.

Results There were 72 incident LN from 1976-2018. Mean age was 38.4 years (SD 16.24), 76\% were female, and 69\% non-Hispanic White. The overall LN incidence between 1976 and 2018 was estimated at 1 per 100,000 (95\%CI 0.8-1.3). The incidence was highest in 30-39 age group. The overall incidence of $\mathrm{LN}$ increased from 0.7 to 1.3 with sex specific incidence in males quadrupling from 0.2 to 0.8 per 100,000 between 1976-1988 and 2000-2018 periods (table 1). LN prevalence increased from 16.8 per 100,000 in 1985 to 21.2 in 2015. LN had SMR of 6.33 (95\%CI 3.81-9.89) compared to the general population. Survival was $50 \%$ at 20 years, and among survivors $40 \%$ had ESRD. There was a widening mortality gap with an increase in the SMR of $7 \%$ per year $(1.07$; 95\%CI 1.03-1.10, $\mathrm{p}<0.001)$ or almost a doubling of the SMR every 10 years (1.90 per 10 years; 95\%CI 1.33-2.70). Conclusions The incidence and prevalence of $\mathrm{LN}$ has increased in the last 40 years. Long-term outcomes in patients with LN remain poor. Patients with $\mathrm{LN}$ had a mortality rate of more than $50 \%$ at 20 years and of those surviving, $40 \%$ had ESRD. The widening mortality gap we observed with near doubling of SMR each decade shows that the mortality reduction in LN patients is not keeping up with the mortality improvements of the general population despite changes in clinical practice over the decades.

\section{RESPONSE GENE TO COMPLEMENT -32 FACILITATES LOCAL RECRUITMENT OF IL-17- PRODUCING CELLS IN IMMUNE COMPLEX MEDIATED GLOMERULONEPHRITIS THROUGH THE CCR6/CCL2O AXIS}

\footnotetext{
1,2Violeta Rus ${ }^{*},{ }^{1}$ Alexandru Tatomir, ${ }^{1,2}$ Vinh Nguyen, ${ }^{1} J o h n$ Papadimitriou, ${ }^{1}$ Sergei Atamas, 1,2 Irina G Luzina, ${ }^{3}$ Tudor Badea, ${ }^{4}$ Sonia Vlaicu, ${ }^{1,2}{ }^{1}$ Horea Rus. ${ }^{1}$ University of Maryland School of Medicine, Baltimore, MD, USA; ${ }^{2}$ VA Maryland Health Care System, Baltimore, MD, USA; ${ }^{3} \mathrm{NIH}$, NEI, Bethesda, MD, USA; ${ }^{4}$ University of Medicine and Pharmacy 'Iuliu Hatieganu', Cluj-Napoca, Romania
}

10.1136/lupus-2021-lupus21century.31

Background Response Gene to Complement (RGC)-32 is a cell cycle regulator induced by complement activation, growth factors and cytokines. RGC-32 mediates TGF- $\beta$ dependent profibrotic pathways, while in immune cells promotes the

Abstract 512 Table 1 Age-adjusted incidence (1976-2018) and prevalence $(1985,1995,2005,2015)$ rates of Lupus Nephritis (LN), 8-county region in Southeast Minnesota.

\begin{tabular}{|c|c|c|c|c|c|c|c|c|c|}
\hline & \multicolumn{5}{|c|}{ LN incidence rates $/ 100,000(95 \% \mathrm{Cl})$ * } & \multicolumn{4}{|c|}{ LN Prevalence rates $/ 100,000(95 \% \mathrm{Cl})$ * } \\
\hline & 1976-1989 & 1990-1999 & $2000-2009$ & $2010-2018$ & $1976-2018$ & 01/01/1985 & 01/01/1995 & $01 / 01 / 2005$ & $01 / 01 / 2015$ \\
\hline Females & $1.2(0.4,2.0)$ & $1.3(0.4,2.2)$ & $1.7(0.7,2.7)$ & $1.7(1.0,2.4)$ & $1.5(1.1,1.9)$ & $25.9(11.4,40.5)$ & $30.7(16.7,44.7)$ & $36.5(22.4,50.7)$ & $33.4(24.7,42.1)$ \\
\hline Males & $0.2(0.0,0.6)$ & $0.0(0.0,0.0)$ & $0.6(0.0,1.2)$ & $0.8(0.4,1.3)$ & $0.5(0.3,0.8)$ & $7.1(0.0,15.1)$ & $6(0.0,13)$ & $1.4(0.0,4.3)$ & $8.6(4.1,13)$ \\
\hline Overall & $0.7(0.2,1.2)$ & $0.6(0.2,1.1)$ & $1.2(0.6,1.8)$ & $1.3(0.9,1.7)$ & $1.0(0.8,1.3)$ & $16.8(8.3,25.3)$ & $18.5(10.7,26.4)$ & $19.4(12.1,26.8)$ & $21.2(16.3,26.2)$ \\
\hline
\end{tabular}

